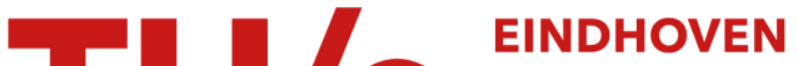 UNIVERSITY OF TECHNOLOGY
}

\section{Limit on suppression of ionization in metastable neon traps due to long-range anisotropy}

Citation for published version (APA):

Doery, M. R., Vredenbregt, E. J. D., op de Beek, S. S., Beijerinck, H. C. W., \& Verhaar, B. J. (1998). Limit on suppression of ionization in metastable neon traps due to long-range anisotropy. Physical Review A : Atomic, Molecular and Optical Physics, 58(5), 3673-3682. https://doi.org/10.1103/PhysRevA.58.3673, https://doi.org/10.1103/PhysRevA.58.3673

DOI:

10.1103/PhysRevA.58.3673

10.1103/PhysRevA.58.3673

Document status and date:

Published: 01/05/1998

\section{Document Version:}

Publisher's PDF, also known as Version of Record (includes final page, issue and volume numbers)

\section{Please check the document version of this publication:}

- A submitted manuscript is the version of the article upon submission and before peer-review. There can be important differences between the submitted version and the official published version of record. People interested in the research are advised to contact the author for the final version of the publication, or visit the $\mathrm{DOI}$ to the publisher's website.

- The final author version and the galley proof are versions of the publication after peer review.

- The final published version features the final layout of the paper including the volume, issue and page numbers.

Link to publication

\section{General rights}

Copyright and moral rights for the publications made accessible in the public portal are retained by the authors and/or other copyright owners and it is a condition of accessing publications that users recognise and abide by the legal requirements associated with these rights.

- Users may download and print one copy of any publication from the public portal for the purpose of private study or research.

- You may not further distribute the material or use it for any profit-making activity or commercial gain

- You may freely distribute the URL identifying the publication in the public portal.

If the publication is distributed under the terms of Article $25 \mathrm{fa}$ of the Dutch Copyright Act, indicated by the "Taverne" license above, please follow below link for the End User Agreement:

www.tue.nl/taverne

Take down policy

If you believe that this document breaches copyright please contact us at:

openaccess@tue.nl

providing details and we will investigate your claim. 


\title{
Limit on suppression of ionization in metastable neon traps due to long-range anisotropy
}

\author{
M. R. Doery, ${ }^{*}$ E. J. D. Vredenbregt, S. S. Op de Beek, H. C. W. Beijerinck, and B. J. Verhaar \\ Physics Department, Eindhoven University of Technology, P.O. Box 513, 5600 MB Eindhoven, The Netherlands
}

(Received 26 May 1998)

\begin{abstract}
This paper investigates the possibility of suppressing the ionization rate in a magnetostatic trap of metastable neon atoms by spin-polarizing the atoms. Suppression of the ionization is critical for the possibility of reaching Bose-Einstein condensation with such atoms. We estimate the relevant long-range interactions for the system, consisting of electric quadrupole-quadrupole and dipole-induced dipole terms, and develop short-range potentials based on the $\mathrm{Na}_{2}$ singlet and triplet potentials. The autoionization widths of the system are also calculated. With these ingredients we calculate the ionization rate for spin-polarized and for spin-isotropic samples, caused by anisotropy of the long-range interactions. We find that spin polarization may allow for four orders of magnitude suppression of the ionization rate for $\mathrm{Ne}$. The results depend sensitively on a precise knowledge of the interaction potentials, however, pointing out the need for experimental input. The same model gives a suppression ratio close to unity for metastable xenon in accordance with experimental results, due to a much increased anisotropy in this case. [S1050-2947(98)03111-4]
\end{abstract}

PACS number(s): 34.50.Fa, 32.80.Pj, 34.50.-s, 34.20.Mq

\section{INTRODUCTION}

The experimental observation of Bose-Einstein condensation (BEC) in 1995 by three different groups [1-3] has fueled renewed interest in this subject, with many more groups trying to achieve the conditions under which the BEC transition occurs. Stated simply, one has to reach the point where the nearest-neighbor distance between the atoms is of the same order of magnitude as their de Broglie wavelength $\Lambda$. In mathematical terms, the condition to fulfill is [4]

$$
n \Lambda^{3} \geqslant 2.61
$$

with $n$ the atomic number density. Experimentally, this can be achieved by evaporative cooling of atoms caught in a trap, with typical final conditions given by a temperature of $T_{c}$ $\approx 1 \mu \mathrm{K}$ and a density of $n \approx 10^{14}$ atoms $/ \mathrm{cm}^{3}$ [1] for $\mathrm{Na}$, and $T_{c} \approx 170 \mathrm{nK}$ at $n \approx 3 \times 10^{12}$ atoms $/ \mathrm{cm}^{3}$ [2] for $\mathrm{Rb}$.

Both the efficiency of the cooling process and the final density achievable depend on the rates of inelastic and elastic collisions in the ultracold atom clouds used [5]. With alkalimetal atoms, inelastic loss in binary collisions between ground-state atoms is due mostly to hyperfine-changing collisions, with rates on the order of $10^{-15} \mathrm{~cm}^{3} / \mathrm{s}$ for $\mathrm{Rb}[6,7]$. These rates are generally low enough to allow condition (1) to be reached [8]. In addition, all the alkali-metal atoms have closed optical transitions from the ground state that are accessible with commercially available lasers, allowing for initial trapping and cooling in a magneto-optical trap (MOT) [9]. As a result, BEC has now been achieved in $\mathrm{Na}, \mathrm{Rb}$, and $\mathrm{Li}$, while work is in progress on $\mathrm{K}$ and $\mathrm{Cs}$.

In comparison, the properties of rare-gas atoms [10] seem less promising. As with atomic hydrogen, laser cooling and trapping starting from the ground state are not practical due to the very short wavelengths required ( $75 \mathrm{~nm}$ for neon). All rare-gas atoms have a metastable first excited state, however,

\footnotetext{
*Present address: Physics Laboratory, National Institute of Standards and Technology, Gaithersburg, MD 20899.
}

that does allow for optical manipulation. For He, this is the $\left\{(1 s 2 s) 2{ }^{3} S\right\}$ state, while for $\mathrm{Ne}(n=2)$ through $\mathrm{Xe}(n$ $=5)$ the appropriate state can be written $\left\{(n p)^{5}(n\right.$ $\left.+1) s^{3} P_{2}\right\}$. Condensates of such atoms might show interesting new phenomena related to the available electronic energy, such as collective decay or ionization. In addition, an " "atom laser" [11] based on such condensates may find applications that are not covered by alkali-metal atoms: because of the large internal energy of metastable rare-gas atoms, one might make the comparison with the high-energy photons of an extreme ultraviolet optical laser as opposed to those from a HeNe laser. On the downside, this internal energy of metastable rare-gas atoms is always enough to allow for ionization in binary collisions [12]. Consequently, ionization becomes a major loss process in metastable atom traps, with experimental rates reported to be as large as 5 $\times 10^{-11} \mathrm{~cm}^{3} / \mathrm{s}$ for $\mathrm{He}\left(2^{3} S\right)$ [13]. Such high rates make achieving condition (1) impractical using the techniques currently employed for alkali-metal atoms.

For He, Shlyapnikov and co-workers $[14,15]$ have shown, however, that the ionization rate can be very strongly suppressed by spin-polarizing the atoms. In the process

$$
\mathrm{He} \uparrow\left(2{ }^{3} S\right)+\operatorname{He} \uparrow\left(2{ }^{3} S\right) \rightarrow \operatorname{He}\left(1{ }^{1} S\right)+\mathrm{He}^{+}\left(1^{2} S\right)+e^{-},
$$

the initial state then has total electron spin $S=2$, while in the final state spin can only be $S=0,1$. Because the ionization process conserves electron spin, ionization is prohibited in the $S=2$ (quintet) states and can proceed only after a magnetic interaction between the electrons causes a spin-flip during a binary encounter. Shlyapnikov and co-workers [14,15] found that at ultracold temperatures the relatively weak spindipole or magnetic spin-spin interaction then limits the ionization rate in spin-polarized metastable He samples to the order of $10^{-14} \mathrm{~cm}^{3} / \mathrm{s}[14,15]$.

For the heavier rare gases $\mathrm{Ne}$ through $\mathrm{Xe}$, the spinconservation rule holds also. Therefore, suppression of ionization by spin polarization should be feasible as well. An added complication, however, is the fact that the unfilled 
TABLE I. Spectroscopic notation, equivalent Russel-Saunders LSJ notation where applicable, atomic energy, and electronic angular momentum quantum number $J$ for the four states of $\mathrm{Ne}$ in the $\left\{(2 p)^{5} 3 s\right\}$ configuration.

\begin{tabular}{lccc}
\hline \hline $\begin{array}{l}\text { Spectroscopic } \\
\text { notation }\end{array}$ & $\begin{array}{c}\text { LSJ } \\
\text { notation }\end{array}$ & $\begin{array}{c}\text { Energy } \\
\left(\mathrm{cm}^{-1}\right)\end{array}$ & $J$ \\
\hline $3 s^{\prime}[1 / 2]_{1}$ & & 135888.7137 & 1 \\
$3 s^{\prime}[1 / 2]_{0}$ & ${ }^{3} P_{0}$ & 134818.6405 & 0 \\
$3 s[3 / 2]_{1}$ & & 134459.2871 & 1 \\
$3 s[3 / 2]_{2}$ & ${ }^{3} P_{2}$ & 134041.8400 & 2 \\
\hline \hline
\end{tabular}

$(n p)^{5}$ core now has $p$ character, as opposed to the He case, where it has $s$ character. This causes an orientation dependence of the electrostatic interaction potentials that is present even at large internuclear separation. During a collision, this causes the $(n p)^{5}$ cores to reorient, which, through the spinorbit interaction, induces electron spin-flips. Since this interaction has an electrostatic origin rather than the magnetic one that causes spin-flips in metastable He collisions, one may expect it to have a much larger influence and possibly limit the ionization rate for spin-polarized samples to much larger values than for He. It is the purpose of this paper to estimate these effects for trapped clouds of atoms.

Out of the various metastable rare gases, we concentrate on metastable neon because this element is being used in experiments presently going on in our laboratory [16]. In addition, the properties necessary for our calculations are most readily available for this species [17]. While for most alkali-metal atoms the properties of the atom-atom interactions are known quite accurately [18], the situation is very different for the metastable rare gases, so that we have to make our own estimates.

\section{METASTABLE NEON IONIZATION RATES}

The $\left\{(n p)^{5}(n+1) s\right\}$ configuration of any of the heavy metastable rare gases contains four energy levels, with the ${ }^{3} P_{2}$ state always the lowest-lying state. Table I lists the properties of these states in neon. The four atomic fine- structure states give rise to a total of 144 molecular states. In what follows we will concentrate on the 25 states that connect asymptotically to $\mathrm{Ne}\left({ }^{3} P_{2}\right)+\mathrm{Ne}\left({ }^{3} P_{2}\right)$, since the ${ }^{3} P_{2}$ state is the only state that is both metastable and has nonzero angular momentum, thus allowing for magnetic trapping and spin polarization.

In the following sections we develop autoionization widths, short-range, and long-range real potentials for the equivalent process of Eq. (2) for $\mathrm{Ne}\left({ }^{3} P_{2}\right)$. We then use them in a standard coupled-channels calculation [19] of the scattering matrix $\mathcal{S}$. For each (positive) initial kinetic energy, we express the asymptotic solution matrix of the calculation as a linear combination of Bessel and Neumann functions for the radial part, multiplied by channel functions $\Psi_{k}$ denoting electronic and angular momentum states. For $\Psi_{k}$ we have used states with well-defined total angular momentum quantum number $P$ with projection $M$ onto a laboratory axis, rotational quantum number $l$, electronic (molecular) angular momentum quantum number $j$, and parity $\Pi$, denoted as

$$
\left|\Psi_{k}\right\rangle=|\Pi j l P M\rangle \text {. }
$$

Since $\Pi, P$, and $M$ are conserved in a collision, this has the advantage that the coupled equations can be separated into blocks characterized by these quantum numbers, which can then be solved separately. The $\mathcal{S}$ matrix for such a block will be denoted $S_{\Pi Р M}$. The spin-polarized and nonpolarized channels can be easily identified because all the nonionizing quintet states have $j=4$ exclusively.

For any given parity, only even $l(\Pi=1)$ or odd $l(\Pi=$ -1) contribute. Because the $\mathrm{Ne}\left({ }^{3} P_{2}\right)$ atoms we are dealing with are bosons, in addition only states that are symmetric under the exchange of two atoms can contribute. This translates into the rule that $l+j$ must be even. Since parity is conserved in a collision, it follows that the nonionizing quintet states $(j=4)$ can only couple to other even $j=0,2$ states.

Because of the presence of ionization, the $\mathcal{S}$ matrix for (in)elastic scattering is not unitary. Ionization rates follow from the loss of flux described by this property. For the case of a trapped cloud of atoms, the distribution of the direction of the initial relative velocity of two atoms is spatially isotropic. Taking this into account, we find for the ionization rate $K^{\mathrm{pol}}$ in a polarized atom sample

$$
\begin{aligned}
K^{\mathrm{pol}} & =\sum_{l=0}^{\infty} K_{l}^{\mathrm{pol}} \\
& =\frac{2 \pi}{9 k^{2}} \sum_{l=0, \text { even }}^{\infty} \sum_{P=|j-l|}^{|j+l|}(2 P+1)\left\{1-\sum_{j^{\prime}} \sum_{l^{\prime}}\left|S_{(\Pi=1) P M}\left(j^{\prime} l^{\prime} \leftarrow j l\right)\right|^{2}\right\},
\end{aligned}
$$

where the electronic angular momentum quantum number is limited to $j=4$. Similarly, the rate for an unpolarized sample is given by

$$
\begin{aligned}
K^{\mathrm{unpol}} & =\sum_{l=0}^{\infty} K_{l}^{\mathrm{unpol}} \\
& =\frac{\pi}{50 k^{2}} \sum_{l=0}^{\infty} \sum_{j=0}^{4}\left[1+(-1)^{l-j}\right]^{2} \sum_{P=|j-l|}^{|j+l|}(2 P+1)\left\{1-\sum_{j^{\prime}} \sum_{l^{\prime}}\left|S_{\left[\Pi=(-1)^{l}\right] P M}\left(j^{\prime} l^{\prime} \leftarrow j l\right)\right|^{2}\right\} .
\end{aligned}
$$


A suppression ratio $\eta$ may then be defined as

$$
\eta=K^{\mathrm{unpol}} / K^{\mathrm{pol}}
$$

\section{LONG-RANGE POTENTIALS}

For two atoms with $\left\{(n p)^{5}(n+1) s\right\}$ configurations the lowest-order terms in the electrostatic interaction are the quadrupole-quadrupole (QQ) and dipole-induced dipole (DD) terms [20,21], with long-range behavior

$$
\begin{aligned}
& V_{\mathrm{QQ}}=C_{5} R^{-5}, \\
& V_{\mathrm{DD}}=C_{6} R^{-6} .
\end{aligned}
$$

The QQ term is due entirely to the quadrupole moment of the tightly bound $(n p)^{5}$ core and is therefore relatively small. Its order of magnitude is given by

$$
C_{5} \approx \frac{q^{2}}{4 \pi \epsilon_{0}}\left\langle r_{h}^{2}\right\rangle^{2}
$$

with $\left\langle r_{h}^{2}\right\rangle$ the expectation value of the square of the orbital radius of the hole state in the $(n p)^{5}$ core and $q$ the electron charge. Using electron wave functions calculated by Hausamann [22] we have found $\left\langle r_{h}^{2}\right\rangle \approx 1.0$ a.u., leading to $C_{5}$ $\approx 1$ a.u. Numerically, we have implemented the complete state dependence of $C_{5}$ based on the theory of Refs. [20,21], evaluated over a basis of product states $\left|\alpha_{1} j_{1}^{\text {at }} m_{1}\right\rangle\left|\alpha_{2} j_{2}^{\text {at }} m_{2}\right\rangle$, where $j^{\text {at }}$ is the atomic electronic angular momentum corresponding to the atomic state labeled by $\alpha$ and $m$ is its projection onto the internuclear axis. The maximum and minimum values of $C_{5}$ that occur in the $\mathrm{Ne}\left({ }^{3} P_{2}\right)+\operatorname{Ne}\left({ }^{3} P_{2}\right)$ subset are 0.47 a.u. and -0.37 a.u., respectively. These values will increase when going to the heavier rare gases Ar, $\mathrm{Kr}$, and $\mathrm{Xe}$, in accordance with the increasing value of the $\left\langle r_{h}^{2}\right\rangle$-matrix element, which we have listed in Table III (taken from Ref. [23]). According to the table, for Xe the QQ term will be about 26 times larger than for $\mathrm{Ne}$.

To obtain an estimate of the DD term, we have used a second-order perturbation treatment of the DD interaction based on experimental values [17] for the $\left\{(n p)^{5}(n+1) s\right\}$ to $\left\{(n p)^{5}(n+1) p\right\}$ transition, analogous to our previous treatment of the DD interaction for the $\left\{(n p)^{5}(n+1) s\right\}$ $+\left\{(n p)^{5}(n+1) p\right\}$ configuration in the rare gases [24]. Here we have included all possible product states of all $\left\{(n p)^{5}(n+1) s\right\}$ and $\left\{(n p)^{5}(n+1) p\right\}$ states, which may limit the accuracy of the resulting $C_{6}$ values to $30-40 \%$ [25]. The orientation dependence of the values obtained in this way can be understood from a semiclassical description of the DD interaction, where the polarizability of a $\mathrm{Ne}$ atom is thought to be made up of a combination of a large, spatially isotropic value $d_{v} \approx 153$ a.u. due essentially to the ( $n$ $+1) s$ valence electron, with an added small term $d_{c}$ $\approx 1.33$ a.u. determined by the polarizability of the core. Only this smaller term depends on the orientation of the $(n p)^{5}$ core, in the way described by Bussery and AubertFrecon [26]. Such a model results in $C_{6}$ values for the product state $\left|\alpha_{1} j_{1}^{\text {at }} m_{1}\right\rangle\left|\alpha_{2} j_{2}^{\text {at }} m_{2}\right\rangle$ that (for $j_{1}^{\text {at }}=j_{2}^{\text {at }}=2$ ) conform to
TABLE II. Hund's case $(c)$ symmetries, $C_{6}$ and $C_{5}$ coefficients of the 25 adiabatic molecular states asymptotically connected to $\mathrm{Ne}\left({ }^{3} P_{2}\right)+\mathrm{Ne}\left({ }^{3} P_{2}\right)$, derived from the $\mathrm{Na}_{2}\left({ }^{1} \Sigma_{g}^{+}\right)$and $\mathrm{Na}_{2}\left({ }^{3} \Sigma_{u}^{+}\right)$potentials with the formalism of Sec. IV. The degeneracy of all $\Omega$ $=0$ states is 1 , that of all others is 2 .

\begin{tabular}{lrc}
\hline \hline$\Omega$ & $C_{5}$ (a.u.) & $C_{6}$ (a.u.) \\
\hline 3 & -0.3700 & -1953.24 \\
2 & -0.2878 & -1951.33 \\
2 & -0.2673 & -1952.60 \\
1 & -0.2028 & -1951.23 \\
$0^{-}$ & -0.1525 & -1951.64 \\
1 & -0.1318 & -1951.13 \\
$0^{+}$ & -0.0679 & -1954.13 \\
3 & 0.1233 & -1953.36 \\
2 & 0.1233 & -1952.73 \\
1 & 0.1617 & -1952.78 \\
1 & 0.1730 & -1953.52 \\
$0^{+}$ & 0.2173 & -1955.58 \\
4 & 0.2467 & -1955.54 \\
$0^{-}$ & 0.3992 & -1951.48 \\
$0^{+}$ & 0.4673 & -1952.38 \\
\hline \hline
\end{tabular}

$$
C_{6} \propto d_{v}^{2}+\frac{1}{18} d_{c} d_{v}\left(34-m_{1}^{2}-m_{2}^{2}\right)
$$

to first order in $d_{c}$, which is borne out by our calculated values. Since $d_{c} \approx 0.01 d_{v}$ for $\mathrm{Ne}$, the magnitude of the orientation-dependent term, 4.5 a.u., is only $0.25 \%$ of the average value $C_{6}=1953$ a.u. Again, this fraction increases when going to heavier rare gases, finally attaining $1.3 \%$ for Xe (see Tables II and III). For He, Eq. (10) is still appropriate for the limiting value $d_{c}=0$.

Table II lists the properties of the 25 molecular eigenstates that diagonalize the combination of the DD- and QQinteraction matrices with the atomic energy-matrix. From the $C_{5}$ and $C_{6}$ values listed, it becomes clear that, on average, the QQ term is quite a small effect. For one, it is overwhelmed by the DD term out to internuclear distances of $R$ $\approx 4000$ a.u. Beyond that point, its value is considerably less than $1 \mathrm{pK}$, far below the collision energy currently obtainable in atom traps. Therefore, the low-energy behavior of the the scattering rates will be determined by the DD term. Since, however, the anisotropy due to the QQ term (1 a.u./ $R^{5}$ ) is of the same order of magnitude as the QQ term itself, while that due to the DD term $\left(4.5\right.$ a.u. $\left./ R^{6}\right)$ is only a fraction of its average value, the QQ term should still be expected to determine the absolute value of the ionization rates for spin-polarized atoms, which are governed by inelastic, spin-changing processes.

\section{SHORT-RANGE POTENTIALS}

As mentioned in the Introduction, there is little information available about the interaction potentials of the heavier metastable rare gases. We can obtain rough estimates of these, however, by considering the analogy with ground-state alkali-metal atoms. For the case of $\operatorname{Ne}\left\{(2 p)^{5} 3 s\right\}$ $+\mathrm{Ne}\left\{(2 p)^{5} 3 s\right\}$, the appropriate choice is to base an estimate 
TABLE III. Atomic and core polarizability, $d_{v}$ and $d_{c}$, respectively, and expectation value of the square of the orbital radius of the hole state in the $(n p)^{5}$ core (taken from Ref. [23]) for metastable rare-gas atoms $\mathrm{Ne}$ through Xe. The atomic polarizability corresponds to the value listed by Ref. [30] for configuration $\left\{(n p)^{5}(n+1) s\right\}$, while the core polarizability corresponds to the ionic configuration $(n p)^{5}$. Since $d_{c} / d_{v} \ll 1$ always, one may equate the polarizability of the $(n+1) s$-valence electron alone with the atomic polarizability. The last two columns give the anisotropy parameters $\Delta C_{6}$ and $\Delta C_{5}$ relative to those for $\mathrm{Ne}\left({ }^{3} P_{2}\right) \quad\left(\Delta C_{5}=0.84\right.$ a.u., $\Delta C_{6}=4.5$ a.u. $)$. For clarity, the table also lists the anisotropy parameters for $\mathrm{He}$ as being zero: in this case the core has a spatially isotropic $(1 s)$ configuration.

\begin{tabular}{lcccccc}
\hline \hline $\begin{array}{l}\text { Rare-gas } \\
\text { atom }\end{array}$ & $\begin{array}{c}\text { Principal quantum } \\
\text { number } n\end{array}$ & $\begin{array}{c}d_{v} \\
(\text { a.u. })\end{array}$ & $\begin{array}{c}d_{c} \\
(\text { a.u. })\end{array}$ & $\begin{array}{c}\left\langle r_{h}^{2}\right\rangle \\
(\text { a.u. })\end{array}$ & $\begin{array}{c}\text { anisotropy } \Delta C_{6} \\
\text { relative to } \mathrm{Ne}\left({ }^{3} P_{2}\right)\end{array}$ & $\begin{array}{c}\text { anisotropy } \Delta C_{5} \\
\text { relative to } \operatorname{Ne}\left({ }^{3} P_{2}\right)\end{array}$ \\
\hline $\mathrm{He}$ & & & & 0 & 0 \\
$\mathrm{Ne}$ & 2 & 153 & 1.33 & 1.23 & 1.0 & 1.0 \\
$\mathrm{Ar}$ & 3 & 305 & 7.90 & 3.31 & 3.0 & 7.3 \\
$\mathrm{Kr}$ & 4 & 363 & 12.8 & 4.46 & 4.1 & 13.2 \\
$\mathrm{Xe}$ & 5 & 511 & 23.1 & 6.28 & 5.2 & 26.1 \\
\hline \hline
\end{tabular}

on the potentials of the system $\mathrm{Na}\left\{(2 p)^{6} 3 s\right\}+\mathrm{Na}\left\{(2 p)^{6} 3 s\right\}$, consisting of the $\mathrm{Na}_{2}\left({ }^{3} \Sigma_{u}^{+}\right)$and $\mathrm{Na}_{2}\left({ }^{1} \Sigma_{g}^{+}\right)$states that have been studied by a number of authors [27].

Following Hennecart et al. [28,29], we can expand each of the four $\operatorname{Ne}\left\{(2 p)^{5} 3 s\right\}$ atomic states (labeled by $\left.\alpha=1, \ldots, 4\right)$ on a basis of single-electron valence (superscript $v$ ) and core states (superscript $c$ ),

$$
\left|\alpha j^{\text {at }} m\right\rangle=\sum_{m_{l}^{c}=-1}^{1} \sum_{m_{s}^{c}=-1 / 2}^{1 / 2} \sum_{m_{s}^{v}=-1 / 2}^{1 / 2} c_{m_{l}^{c}, m_{s}^{c}, m_{s}^{v}}^{\alpha}\left|\left(l^{c}=1\right) m_{l}^{c}\right\rangle\left|\left(s^{c}=\frac{1}{2}\right) m_{s}^{c}\right\rangle\left|\left(l^{v}=0\right)\left(m_{l}^{v}=0\right)\right\rangle\left|\left(s^{v}=\frac{1}{2}\right) m_{s}^{v}\right\rangle .
$$

In the following we will write $\left|0^{v}\right\rangle$ as short notation for $\left|\left(l^{v}=0\right)\left(m_{l}^{v}=0\right)\right\rangle$ and leave out quantum numbers that do not change. If we write the products of the valence electron states in terms of triplet and singlet states,

$$
\begin{gathered}
\left|T_{+1}\right\rangle=\left|0^{v}\right\rangle_{1}\left|0^{v}\right\rangle_{2}\left|m_{s}^{v}=+\frac{1}{2}\right\rangle_{1}\left|m_{s}^{v}=+\frac{1}{2}\right\rangle_{2}, \\
\left|T_{-1}\right\rangle=\left|0^{v}\right\rangle_{1}\left|0^{v}\right\rangle_{2}\left|m_{s}^{v}=-\frac{1}{2}\right\rangle_{1}\left|m_{s}^{v}=-\frac{1}{2}\right\rangle_{2}, \\
\left|T_{0}\right\rangle=\frac{1}{\sqrt{2}}\left|0^{v}\right\rangle_{1}\left|0^{v}\right\rangle_{2} \times\left(\left|m_{s}^{v}=+\frac{1}{2}\right\rangle_{1}\left|m_{s}^{v}=-\frac{1}{2}\right\rangle_{2}\right. \\
\left.\quad+\left|m_{s}^{v}=-\frac{1}{2}\right\rangle_{1}\left|m_{s}^{v}=+\frac{1}{2}\right\rangle_{2}\right), \\
|S\rangle=\frac{1}{\sqrt{2}}\left|0^{v}\right\rangle_{1}\left|0^{v}\right\rangle_{2} \times\left(\left|m_{s}^{v}=+\frac{1}{2}\right\rangle_{1}\left|m_{s}^{v}=-\frac{1}{2}\right\rangle_{2}\right. \\
\left.-\left|m_{s}^{v}=-\frac{1}{2}\right\rangle_{1}\left|m_{s}^{v}=+\frac{1}{2}\right\rangle_{2}\right),
\end{gathered}
$$

then the $\mathrm{Na}_{2}$ potentials correspond to matrix elements of the atom-atom interaction $V_{\mathrm{Na}}$ given by

$$
\begin{gathered}
\mathrm{Na}_{2}\left({ }^{3} \Sigma_{u}^{+}\right):\left\langle T_{i}\left|V_{\mathrm{Na}}\right| T_{j}\right\rangle \delta_{i, j} \quad(i, j=-1,0,1), \\
\mathrm{Na}_{2}\left({ }^{1} \Sigma_{g}^{+}\right):\left\langle S\left|V_{\mathrm{Na}}\right| S\right\rangle .
\end{gathered}
$$

From this we can construct $\mathrm{Ne}_{2}$ potentials if we multiply $V_{\mathrm{Na}}$ by a unit core-factor that is diagonal in all core quantum numbers. Such potentials should contain the essential features of the valence-electron exchange interactions, but do not describe the interaction of the core hole of one atom with the valence electron of the other. Since the radius of the valence electron's orbital is considerably larger than that of the core electron's [30], this approximation should be reasonable. Also, core-valence electron terms can be added in a semiempirical way. For an accurate description of the wells and turning points, however, either $a b$ initio potentials or experimental data would be required.

Figure 1 displays diagonalized $\mathrm{Ne}\left({ }^{3} P_{2}\right)+\mathrm{Ne}\left({ }^{3} P_{2}\right)$ potentials developed in this way, together with the $\mathrm{Na}_{2}\left({ }^{1} \Sigma_{g}^{+}\right)$and $\mathrm{Na}_{2}\left({ }^{3} \Sigma_{u}^{+}\right)$potentials on which they are based. The nine possible fully spin-polarized $\mathrm{Ne}_{2}$ states that are characterized both by a total angular momentum $j=4$ and a total electron spin $S=2$ exactly coincide with the $\mathrm{Na}_{2}\left({ }^{3} \Sigma_{u}^{+}\right)$states. The

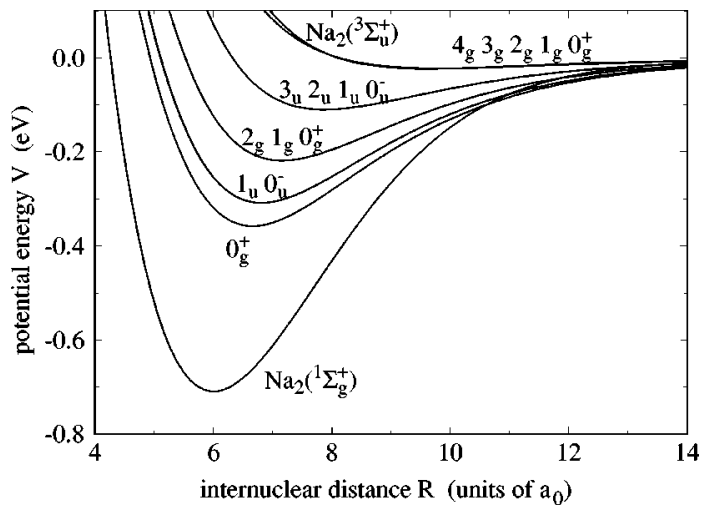

FIG. 1. The adiabatic potentials of $\mathrm{Na}_{2}\left({ }^{3} \Sigma_{u}^{+}\right)$and $\mathrm{Na}_{2}\left({ }^{1} \Sigma_{g}^{+}\right)$ [27] and the short-range, adiabatic, $\operatorname{Ne}\left({ }^{3} P_{2}\right)+\operatorname{Ne}\left({ }^{3} P_{2}\right)$ potentials derived from them with the formalism of Sec. IV. The potentials of the $\mathrm{Ne}_{2}$ quintet states (characterized by $j=4$ and $S=2$ ) coincide with those of $\mathrm{Na}_{2}\left({ }^{3} \Sigma_{u}^{+}\right)$. The molecular potentials are labeled by their Hund's case $(c)$ classification. 
repulsion of the valence electrons leads to a turning point $R_{\mathrm{tp}} \approx 8$ a.u. for vanishing asymptotic kinetic energy in this case.

All states conform to Hund's case (c) [31], and in addition can be characterized by their total angular momentum $j$ $=0, \ldots, 4$. Consequently, their $g / u$ symmetry follows immediately from $j$ as $(-1)^{j}$. The well depth of these $\mathrm{Ne}_{2}$ states increases with decreasing $j$, since more and more $\mathrm{Na}_{2}\left({ }^{1} \Sigma_{g}^{+}\right)$character becomes mixed in. The state with the deepest well, of symmetry $0_{g}^{+}$, does not coincide with the $\mathrm{Na}_{2}\left({ }^{1} \Sigma_{g}^{+}\right)$state since it is not a pure singlet. In order for deeper wells to occur, the spin-orbit coupling of each of the $\mathrm{Ne}$ atoms must be allowed to compete with the $\mathrm{Na}_{2}\left({ }^{1} \Sigma_{g}^{+}\right)$$\mathrm{Na}_{2}\left({ }^{3} \Sigma_{u}^{+}\right)$splitting, which requires mixing with states correlating to other asymptotes than $\mathrm{Ne}\left({ }^{3} P_{2}\right)+\mathrm{Ne}\left({ }^{3} P_{2}\right)$ from within the same configuration $\left\{(2 p)^{5} 3 s\right\}+\left\{(2 p)^{5} 3 s\right\}$. We will discuss possible consequences of including only the $\mathrm{Ne}\left({ }^{3} P_{2}\right)+\mathrm{Ne}\left({ }^{3} P_{2}\right)$ configuration in Sec. VIII.

\section{AUTOIONIZATION WIDTHS}

In scattering calculations, ionization can be introduced by adding an imaginary part to the real-valued atomic interaction potentials $[12,32]$, creating a complex "optical" potential $W$,

$$
W(R)=V(R)-i \Gamma(R) / 2,
$$

with $\Gamma(R)$ the so-called autoionization width.

Op de Beek et al. $[33,34]$ have described a numerical method for the calculation of $\Gamma(R)$, based on a recurrence recipe developed by Rico et al. [35]. Briefly, one calculates matrix elements between initial and final states of the Coulomb interaction between the individual electrons involved in the ionization process. In the process considered here,

$$
\begin{aligned}
\mathrm{Ne}\left({ }^{3} P_{2}\right)+\mathrm{Ne}\left({ }^{3} P_{2}\right) \rightarrow & \mathrm{Ne}\left({ }^{1} S\right)+\mathrm{Ne}^{+}\left\{(2 p)^{5}\left({ }^{2} P\right)\right\}+e^{-} \\
& +11.7 \mathrm{eV}
\end{aligned}
$$

the dominant ionization mechanism is the so-called exchange mechanism [36]. This means that the valence electron of atom 1 transfers to the available core state of atom 2, leaving the core of atom 1 to form a positive ion. The valence electron of atom 2 is now no longer bound and leaves the scene. On the basis of valence electron and core states introduced in Eq. (11), a partial ionization amplitude $\gamma$ may be defined, given by

$$
\begin{aligned}
\gamma= & \int d \vec{r}_{1} d \vec{r}_{2} \Psi_{1}^{v}\left(\vec{r}_{1}\right) \Psi_{2}^{c}\left(\vec{r}_{1}\right)^{*} \\
& \times \frac{q^{2}}{4 \pi \epsilon_{0}\left|\vec{r}_{1}-\vec{r}_{2}\right|} \Psi_{2}^{v}\left(\vec{r}_{2}\right) \Psi^{f}\left(\vec{r}_{2}\right)^{*}
\end{aligned}
$$

with $\vec{r}_{1}$ and $\vec{r}_{2}$ the position vectors of the active electrons of atom 1 and 2, respectively. The various single-electron valence and core states can be characterized by their orbital and spin quantum numbers with corresponding projections on the internuclear axis,

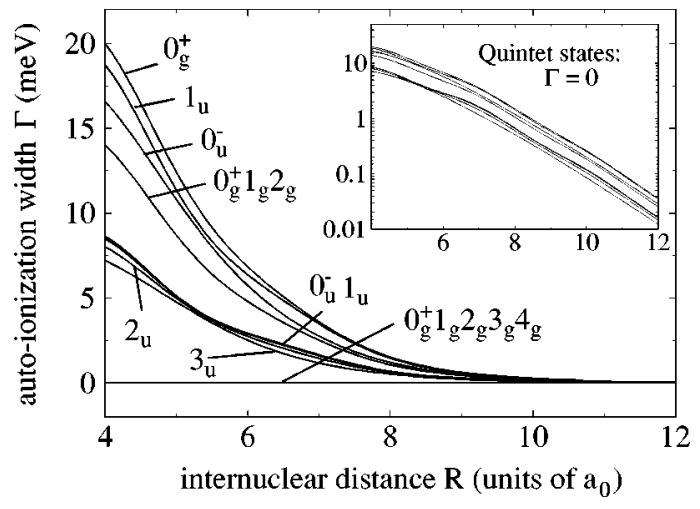

FIG. 2. Ab initio autoionization widths $\Gamma$ for the $\mathrm{Ne}\left({ }^{3} P_{2}\right)+$ $\mathrm{Ne}\left({ }^{3} P_{2}\right)$ system as a function of internuclear distance $R$. The widths are given in the same, adiabatic basis as used for Fig. 1. The inset shows the widths on a logarithmic scale. Note the vanishing widths of the quintet states.

$$
\begin{gathered}
\Psi_{1}^{v} \propto\left|l_{1}^{v}=0 ;\left(m_{l}\right)_{1}^{v}=0\right\rangle\left|s_{1}^{v}=\frac{1}{2} ;\left(m_{s}\right)_{1}^{v}\right\rangle, \\
\Psi_{2}^{v} \propto\left|l_{2}^{v}=0 ;\left(m_{l}\right)_{2}^{v}=0\right\rangle\left|s_{2}^{v}=\frac{1}{2} ;\left(m_{s}\right)_{2}^{v}\right\rangle, \\
\Psi_{2}^{c} \propto\left|l_{2}^{c}=1 ;\left(m_{l}\right)_{2}^{c}\right\rangle\left|s_{2}^{c}=\frac{1}{2} ;\left(m_{s}\right)_{2}^{c}\right\rangle,
\end{gathered}
$$

while the free electron's wave function $\Psi^{f}$ is given by a Coulomb wave, characterized by orbital angular momentum quantum number $\lambda$ with projection $\mu$, multiplied by a spin state $\left|s_{f}=\frac{1}{2} ; m_{f}^{s}\right\rangle$. Since the Coulomb interaction does not affect spin, Eq. (20) implies a $\delta$ function requiring $\left(m_{s}\right)_{1}^{v}$ $+\left(m_{s}\right)_{2}^{v}=\left(m_{s}\right)_{2}^{c}+m_{s}^{f}$. In addition, conservation of the azimuthal part of the orbital angular momentum requires $\left(m_{l}\right)_{1}^{v}+\left(m_{l}\right)_{2}^{v}=\left(m_{l}\right)_{2}^{c}+\mu^{f}$ so that $|\mu| \leqslant 1$.

By using the inverse of the expansion in Eq. (11) on atom 1 as well as atom 2, $\gamma$ may be transformed into a molecular basis. From the resulting molecular ionization amplitudes $\gamma^{\mathrm{mol}}$, matrix elements of the ionization width $\Gamma(R)$ can then be constructed by summing over the available final states (represented symbolically by $F$ ),

$$
\Gamma(R)_{i, j}=\frac{2 \pi}{\sqrt{2 E_{e} \hbar^{2} / m_{e}}} \sum_{F} \gamma_{i, F}^{\mathrm{mol}} \gamma_{j, F}^{\mathrm{mol}}
$$

where $i, j$ denote individual molecular states, $m_{e}$ is the electron mass, and $E_{e}$ is the energy carried away by the electron $\left[E_{e}=11.7 \mathrm{eV}\right.$ for the process of Eq. (19)].

We have calculated all elements of $\Gamma(R)$ relevant for the ionization process of Eq. (19), using electron wave functions tabulated by Hausamann [22], and using a modified version of the computer code developed by Op de Beek et al. $[33,34]$. Figure 2 displays the results in the same basis as used for Fig. 1, for internuclear distances $4<R$ (a.u.) $<12$. As expected, the quintet states all have zero widths. Also as one would expect, the widths of the other states show an approximately exponentially decreasing behavior with $R$. At each $R$, the ionization width is largest for the states with the largest $(S=0)$ component. 


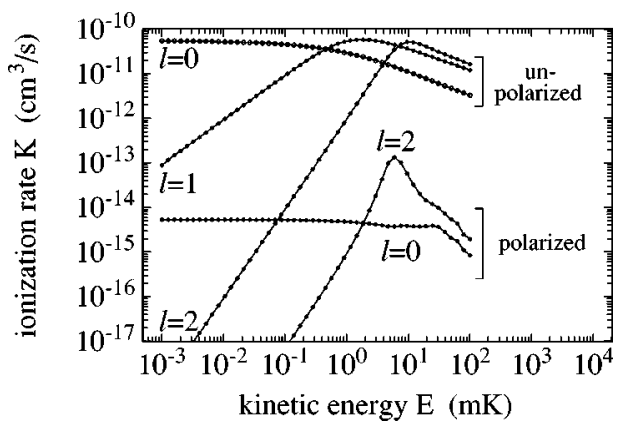

FIG. 3. Partial ionization rates for trapped $\mathrm{Ne}\left({ }^{3} P_{2}\right)$ atoms as a function of the collision energy $E$. Rates are given for unpolarized $\left(K_{l}^{\mathrm{unpol}}\right)$ as well as for polarized atoms $\left(K_{l}^{\mathrm{pol}}\right)$ for angular momentum quantum numbers $l=0, \ldots, 2$. The rates show an obvious Wigner-law behavior. The rates for polarized atoms are about $10^{4} \times$ lower than those for unpolarized atoms.

\section{CALCULATED IONIZATION RATES FOR METASTABLE NEON}

\section{A. Threshold behavior}

With the ingredients developed above, ionization rates $K_{l}$ for polarized and unpolarized atom clouds were calculated as a function of the collision energy $E$ at ultracold temperatures. Figure 3 shows the result for $l=0, \ldots, 2$ in the range $1 \mu \mathrm{K}<E<100 \mathrm{mK}$. The curves show the typical behavior expected from Wigner's threshold law [19] for an exothermic process, $K_{l} \propto E^{l}$. At very low temperature, the contribution for $l=0$ always dominates, leading to an essentially constant ionization rate in this regime. As discussed in Sec. II, due to Bose symmetry, curves for odd partial waves are absent in the case of polarized atoms.

The calculations of Fig. 3 show that a strong suppression, $\eta \approx 10^{4}$, is still possible notwithstanding the long-range anisotropy of the potentials. The resulting suppressed ionization rate, $K^{\mathrm{pol}} \approx 5 \times 10^{-15} \mathrm{~cm}^{3} / \mathrm{s}$ at $E=1 \mu \mathrm{K}$, would certainly allow one to reach the BEC transition, being comparable to the depolarization rate for $\mathrm{Rb}, K^{\mathrm{depol}}=1$ $\times 10^{-15} \mathrm{~cm}^{3} / \mathrm{s}[6,7]$. This result is largely due to the fact that the depolarization due to the long-range anisotropy is counteracted strongly at short range by the separation of the nonionizing quintet states from the other, ionizing states (see

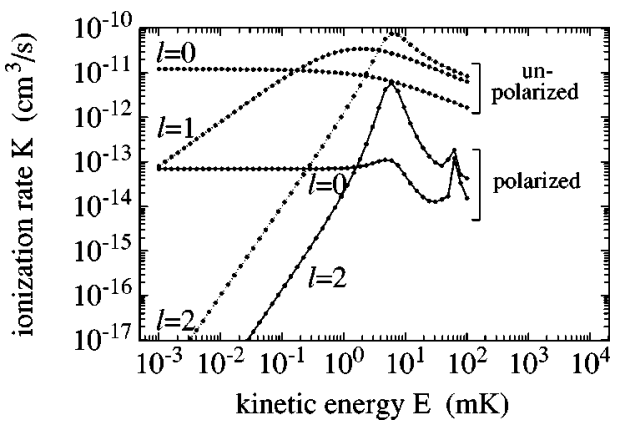

FIG. 4. Partial ionization rates for trapped $\mathrm{Ne}\left({ }^{3} P_{2}\right)$ atoms as a function of the collision energy $E$. In evaluating the potentials for the system, the $\mathrm{Na}_{2}\left({ }^{1} \Sigma_{g}^{+}\right)$potential was artificially replaced by the $\mathrm{Na}_{2}\left({ }^{3} \Sigma_{u}^{+}\right)$potential, removing the large energy splitting between ionizing and nonionizing states. The resulting suppression ratio is much less than for the case of Fig. 3.

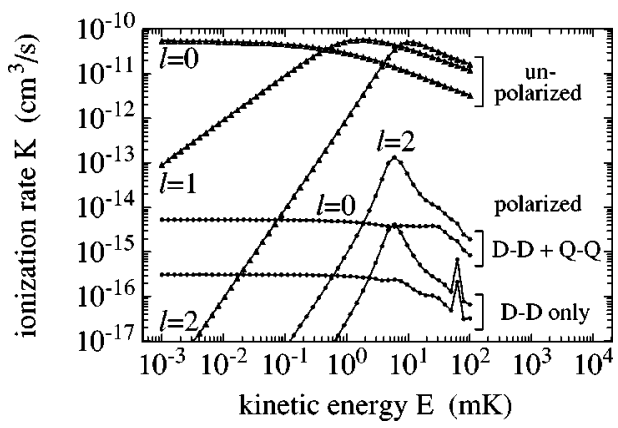

FIG. 5. Partial ionization rates for trapped $\mathrm{Ne}\left({ }^{3} P_{2}\right)$ atoms as a function of the collision energy $E$, calculated including or excluding the effect of the quadrupole-quadrupole (QQ) interaction on the potentials. For the polarized case the results are labeled "DD + QQ' and "DD only," respectively. In general, these rates drop by about an order of magnitude when the QQ term is omitted. For the unpolarized case, the solid lines give the result including the QQ term, and the triangles give the result excluding the QQ term. Little difference is observed in this case.

Fig. 1). This is illustrated in Fig. 4, which displays the rates that result from artificially replacing the $\mathrm{Na}_{2}\left({ }^{1} \Sigma_{g}^{+}\right)$potential by the one for $\mathrm{Na}_{2}\left({ }^{3} \Sigma_{u}^{+}\right)$in the calculation of the $\mathrm{Ne}\left({ }^{3} P_{2}\right)+$ $\mathrm{Ne}\left({ }^{3} P_{2}\right)$ potentials. In this case, no short-range separation between ionizing and nonionizing states occurs, with the result that $\eta$ is reduced to $\eta \approx 170$. This shows that the shortrange behavior of the potentials is very important for the amount of suppression achievable.

To investigate the relative importance of the quadrupolequadrupole versus the dipole-dipole interaction, we have calculated the ionization rates for polarized and nonpolarized samples, with and without the QQ term in the long-range potentials. Figure 5 shows the results. From this figure, it becomes clear that the QQ term is the dominating influence on the ionization rate of spin-polarized samples, which change by about an order of magnitude when the QQ term is removed. At the same time, however, removing the quadrupole term has little influence on the threshold behavior of the rates nor on the ionization rate for unpolarized samples, confirming the dominating role of the dipole term on the elastic scattering already anticipated in Sec. III.

\section{B. Influence of quasibound states}

Since the short-range potentials for the Ne system are not well known, we have to consider variations of the ionization rates due to the uncertainty in the potentials. Of particular importance are resonances due to quasibound states with total energy equal to that of the $l=0$ quintet state. These can give rise to the formation of a relatively long-lived collisional complex, in which the atoms undergo a number of vibrations before they exit. In such a case, the turning point at small $R$, where ionization is most likely, may be encountered several times, leading to an enhanced ionization probability. To investigate the importance of such resonances, we have added a Gaussian "bump" of variable height to the bottom of the well of either the $\mathrm{Na}_{2}\left({ }^{3} \Sigma_{u}^{+}\right)$or the $\mathrm{Na}_{2}\left({ }^{1} \Sigma_{g}^{+}\right)$ potentials from which the $\mathrm{Ne}\left({ }^{3} P_{2}\right)+\mathrm{Ne}\left({ }^{3} P_{2}\right)$ potentials are calculated. By increasing the height of the bump, we decrease the total semiclassical phase $\phi$ available in the well, 


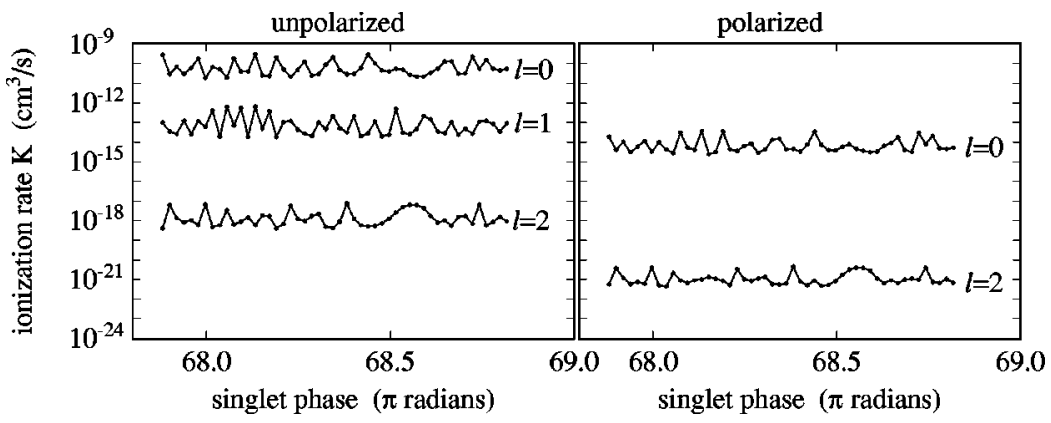

FIG. 6. Partial ionization rates for trapped $\left.\mathrm{Ne}^{3}{ }^{3} P_{2}\right)$ atoms as a function of the depth of the $\mathrm{Na}_{2}\left({ }^{1} \Sigma_{g}^{+}\right)$potential from which the potentials of the $\mathrm{Ne}\left({ }^{3} P_{2}\right)+\operatorname{Ne}\left({ }^{3} P_{2}\right)$ system are calculated. The depth of the $\mathrm{Na}_{2}\left({ }^{1} \Sigma_{g}^{+}\right)$potential is given in terms of the semiclassical phase $\phi$ [see Eq. (23)]. Only relatively small variations in the rates are observed, indicating the minor influence of quasibound resonances in the ionizing states on the ionization rate of the nonionizing, polarized quintet states. The collision energy is $E=1 \mu \mathrm{K}$.

effectively moving the rovibrational states it contains up in energy. The phase $\phi$ is calculated from [37]

$$
\phi=\int_{R_{\mathrm{tp}}}^{\infty} d R \sqrt{-2 \mu V(R) / \hbar^{2}}
$$

with $V(R)$ the potential under consideration and $R_{\mathrm{tp}}$ its classical turning point for vanishing asymptotic kinetic energy. Figures 6 and 7 display the variation of $K_{l}^{\mathrm{pol}}$ and $K_{l}^{\mathrm{unpol}}$ with the phase content of the $\mathrm{Na}_{2}\left({ }^{1} \Sigma_{g}^{+}\right)$and $\mathrm{Na}_{2}\left({ }^{3} \Sigma_{u}^{+}\right)$potentials obtained in this way. Since we vary the phase content by more than $\pi$, resonances are certainly present in both cases.

Figure 6 shows that a variation of the $\mathrm{Na}_{2}\left({ }^{1} \Sigma_{g}^{+}\right)$singlet potential only has a small effect on $K^{\mathrm{pol}}$. In this case, only the energy levels in the quintet states are unaffected by the change in phase content, since they derive solely from the $\mathrm{Na}_{2}\left({ }^{3} \Sigma_{u}^{+}\right)$triplet potential. We can conclude, therefore, that resonances in states other than the quintet states do not seriously alter $K^{\mathrm{pol}}$. The most likely explanation for this is that such resonances are extremely wide, due to the large ionization probability in the nonquintet states, and therefore correspondingly weak. In contrast, a variation of the $\mathrm{Na}_{2}\left({ }^{3} \Sigma_{u}^{+}\right)$ potential depth has a very large effect on $K^{\mathrm{pol}}$, as found from Fig. 7. Here, atoms that initially have $l=0$ in the quintet are coupled to an $l \neq 0$ quintet state at the same total energy, which has a centrifugal barrier at $R \approx 75$ a.u. Such a quasibound level may have a very sharp and therefore strong resonance, since in this state, too, ionization is now prohibited.
During its strongly enhanced lifetime, the molecule may therefore make many vibrations before it tunnels through the barrier or is transferred back to the initial state. All the while, the anisotropy present in the electrostatic interactions weakly couples the molecule to the ionizing states, leading to a greatly enhanced $K^{\mathrm{pol}}$. In our calculations, $K_{l=0}^{\mathrm{pol}}$ reaches a maximum value of $5 \times 10^{-11} \mathrm{~cm}^{3} / \mathrm{s}$ at resonance, at which point $\eta \approx 1$. Again, these values would be prohibitive for reaching the BEC transition. Over the whole phase variation $\Delta \phi=\pi$, however, the suppression ratio mostly takes values between $10^{3}$ and $10^{4}$, showing that a large suppression is rather likely.

These results clearly indicate the extreme importance of an accurate knowledge of the full potentials, or, equivalently, the scattering lengths for the process under consideration. We expect that such information will only be obtained reliably from an $a b$ initio theoretical treatment of the short-range potentials in combination with experimental spectroscopic determinations of the bound molecular states. Until now, this information has only been available for some of the alkalimetal atoms. Since all metastable rare-gas atoms other than He have more than one bosonic isotope, one may hope to avoid such resonances by an appropriate choice of isotope. Since there are a total of nine quintet states, however, with as many as five different potential curves if additional shortrange interactions are considered, there may be so many different resonances that avoiding them altogether is impossible.

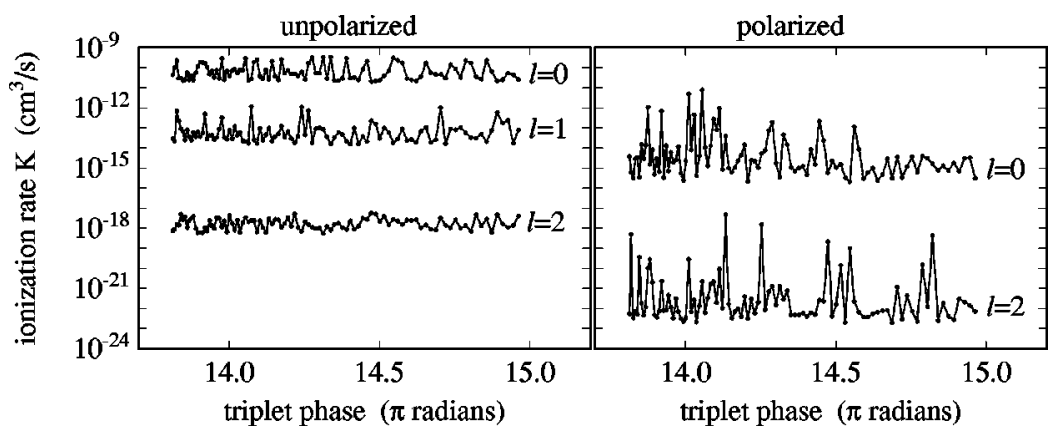

FIG. 7. Partial ionization rates for trapped $\mathrm{Ne}\left({ }^{3} P_{2}\right)$ atoms as a function of the depth of the $\mathrm{Na}_{2}\left({ }^{3} \Sigma_{u}^{+}\right)$potential from which the potentials of the $\mathrm{Ne}\left({ }^{3} P_{2}\right)+\operatorname{Ne}\left({ }^{3} P_{2}\right)$ system are calculated. The depth of the $\mathrm{Na}_{2}\left({ }^{3} \Sigma_{u}^{+}\right)$potential is given in terms of the semiclassical phase $\phi$ [see Eq. (23)]. Very large variations in the rates are observed, indicating narrow quasibound resonances occurring in the nonionizing quintet states. These may adversely affect the possiblities of achieving BEC with metastable rare gases. The collision energy is $E=1 \mu \mathrm{K}$. 


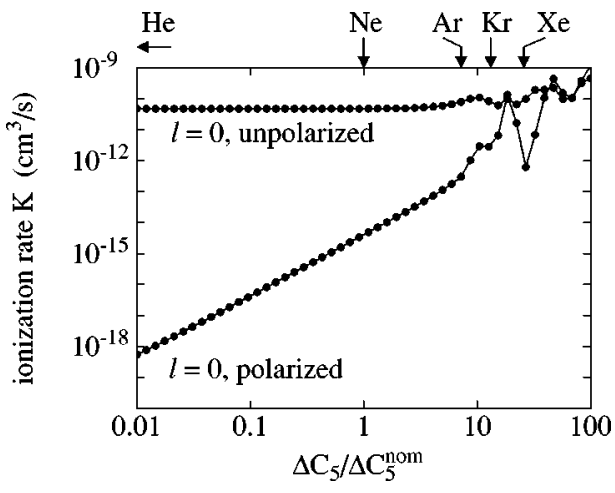

FIG. 8. Partial ionization rates for trapped $\mathrm{Ne}\left({ }^{3} P_{2}\right)$ atoms as a function of the normalized long-range anisotropy parameter $\Delta C_{5} / \Delta C_{5}^{\text {nom }}$. A quadratic dependence of the rate for polarized atoms on the anisotropy parameter is observed. Arrows indicating the estimated anisotropy parameter for metastable $\mathrm{He}, \mathrm{Ne}$, through Xe (listed in Table III) illustrate the decrease in ionization suppression ratio with increasing rare-gas atom mass. The collision energy is $E=1 \mu \mathrm{K}$.

\section{Variation with long-range anisotropy}

In order to make estimates of the suppression ratio for the heavier rare gases Ar through $\mathrm{Xe}$, we have to study the dependence of the ionization rates on long-range anisotropy. According to Table III, both the anisotropy in the dipoledipole term as well as that in the quadrupole-quadrupole term becomes progressively larger going from $\mathrm{Ne}$ to $\mathrm{Xe}$.

A convenient way of representing the variation in $C_{5}$ is by introducing a parameter $\Delta C_{5}=C_{5}^{\max }-C_{5}^{\min }$ equal to the difference of the $C_{5}$ coefficients of the most and least attractive long-range potentials. Table II gives $\Delta C_{5}=0.84$ a.u. for $\mathrm{Ne}$, which may be considered its "nominal" value, which we will denote by $\Delta C_{5}^{\text {nom }}$. We can then study the influence of long-range anisotropy by calculating the ionization rates as a function $\Delta C_{5} / \Delta C_{5}^{\text {nom }}$. Such a calculation is shown in Fig. 8, where, for clarity, we have omitted any anisotropy in the DD term by using an identical, averaged $C_{6}$ coefficient for all states involved. The figure points to a quadratic dependence of $K^{\mathrm{pol}}$ on the dominating anisotropy, while $K^{\text {unpol }}$ is basically unaffected by the change in $C_{5}$. This result can be explained by simple qualitative arguments. The anisotropic long-range interaction $C_{5} / R^{5}$ will cause a rotation of the molecule's electronic angular momentum $j$ over an angle that is proportional to the variation $\Delta C_{5}$ in $C_{5}$, as follows from the equation of motion $I d \Omega / d t=\tau$ for the classical rotation angle $\Omega$ of an object with a moment of inertia $I$ acted upon by a torque $\tau$ [for an anisotropic potential, $\tau$ is proportional to $d V(R) / d \Omega$ ]. In a binary collision of two polarized atoms, by analogy the initially pure quintet state will develop a projection $|P\rangle$ proportional to $\Delta C_{5}$ on other, ionizing states. Since the probability for ionization in such a collision will be proportional to $\langle P \mid P\rangle$, we obtain the quadratic dependence of $K^{\mathrm{pol}}$ on $\Delta C_{5}$ found in Fig. 8 .

From this figure we can therefore conclude that the limit on the suppression ratio set by long-range anisotropy quickly becomes stricter as the rare gas becomes heavier, because $\Delta C_{5}$ is proportional to the square of the quadrupole moment of the core [see Eq. (10)]. We have listed the corresponding matrix elements in Table III for He through Xe, based on values taken from Ref. [23], and indicated the relative anisotropies of all the rare gases in Fig. 8. For He, $\Delta C_{5}=0$ since only $s$ electrons are involved so that no limit on $\eta$ results from the long-range anisotropy. (For He, therefore, a first limit is set by the spin-dipole interaction [14,15]). For Ar through $\mathrm{Xe}$, however, $\Delta C_{5}$ is appreciably larger than for $\mathrm{Ne}$, suggesting that much smaller suppression ratios should be expected for these heavier atoms. Indeed, the available experimental evidence for metastable $\mathrm{Kr}$ [38] and $\mathrm{Xe} \mathrm{[39],}$ with, according to Table III, approximately 200 and 700 times larger $\Delta C_{5}^{2}$ than $\mathrm{Ne}$, respectively, showed basically no suppression at all.

\section{COMPARISON WITH METASTABLE XENON EXPERIMENTS}

The most extensive set of data is available for $\mathrm{Xe}$, where recent experiments [39] suggest that spin polarization does not lead to suppression of the ionization rate for any of the available isotopes; for all bosonic isotopes, spin polarization even seems to increase the ionization rate. From the discussion in Sec. VI C one would already expect $\eta$ to be less than 10 in this case. However, additional dynamical effects due to the larger mass of $\mathrm{Xe}$, and the difference in short-range potentials compared to $\mathrm{Ne}$, should also be taken into account. These changes can be easily incorporated into our model by substituting Cs potentials [40] for the $\mathrm{Na}$ potentials used for $\mathrm{Ne}$ and using the appropriate mass. We have used the same autoionization widths as for $\mathrm{Ne}$, however, since autoionization widths for $\mathrm{Xe}$ are not available.

Figure 9 shows the result of such a treatment for a colli-

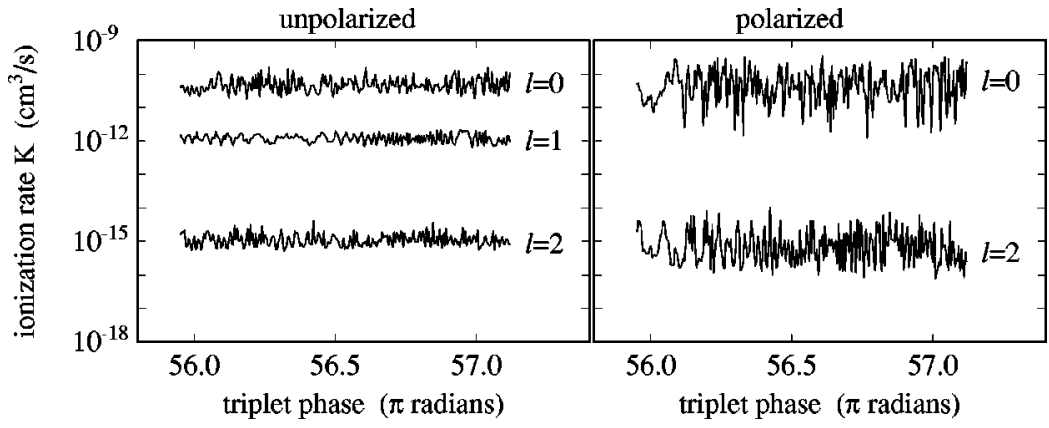

FIG. 9. Same as Fig. 7 but for Xe instead of Ne, replacing the Ne mass by that of Xe, and the Na potentials by those of Cs. The larger anisotropy in the case of Xe leads to much greater polarized ionization rates than for $\mathrm{Ne}$, often exceeding those for unpolarized atoms. A suppression $\eta=10$ is possible, but values $\eta<1$ are more likely. The collision energy is $E=1 \mu \mathrm{K}$. 
sions energy of $1 \mu \mathrm{K}$. Again we varied the amount of phase available in the triplet potential to obtain the variation of the ionization rates for polarized and unpolarized clouds of Xe atoms. Indeed, suppression by a factor of $\eta \approx 10$ is still possible, but over $60 \%$ of the $\phi$ range in Fig. $9, \eta<1$. In fact, the $\phi$-averaged value is now $\langle\eta\rangle=0.7$, showing that for Xe ionization suppression by spin polarization is very unlikely. Here, the QQ term mixes up the polarized and unpolarized states so thorougly at long range that they basically ionize at the same rate. $\eta<1$ can then be considered a quantumstatistical effect: for polarized atoms, the $l=0$ partial wave necessarily represents a greater flux than for unpolarized atoms, since in the former case the $l=1$ partial wave is absent.

\section{DISCUSSION}

In this paper we have developed a semiquantitative description of ionization in metastable neon collisions at very low temperatures. In fact, this description holds for all the heavy metastable rare-gas atoms, but we have concentrated on neon because of ongoing experiments in our laboratory as well as the availability of the properties needed as input to the model. We have put special emphasis on obtaining a lower limit on the ionization rate for spin-polarized atoms, in order to investigate the possibilites of reaching Bose-Einstein condensation with metastable rare-gas atoms. While we are not able to provide a definitive answer, we do provide a framework of potentials and autoionization widths that allow one to investigate the system in a qualitative way. It has become clear that such a limit depends strongly on the occurrence of quasibound resonances in the potentials for fully polarized atoms with $l>0$. While it is possible to obtain reasonable estimates of the anisotropy of the long-range part of these potentials, sufficiently accurate information on the short-range parts is not available at this moment. Such potentials may become available in the near future, however [41]. Our calculations show that, under favorable conditions, the long-range anisotropy still allows for very effective suppression (up to $10^{4}$ times) of ionization by spin polarization, at least for metastable Ne. This suppression becomes less effective as the atom becomes heavier, with a limit of $\eta$ $\approx 10$ for Xe.

Additional couplings may exist between the quintet states and the ionizing states that we have not considered. Such couplings may occur at smaller $R$, where core-valence interactions become more important. The interactions that will have an effect on the ionization rate for spin-polarized atoms must create a direct coupling between a quintet state and other, ionizing states. For Xe, e.g., one should expect a large effect of the second-order spin-orbit splitting from the experience with Cs $[6,7]$. A simple splitting of the various quintet potentials, however, due to a dependence of the potentials on a $\sigma$ or $\pi$ orientation of the cores, does not have such an effect, at least not for small magnetic confining fields. This is because metastable rare-gas atoms in general lack hyperfine structure, so that exothermic exit channels do not exist. As a result, depolarization through such a mechanism is prohibited. We have checked this by adding a phenomenological $\sigma / \pi$ core splitting to our potentials, with an order of magnitude comparable to that found in the $\mathrm{Ne}+\mathrm{Ne}^{+}$system [42]

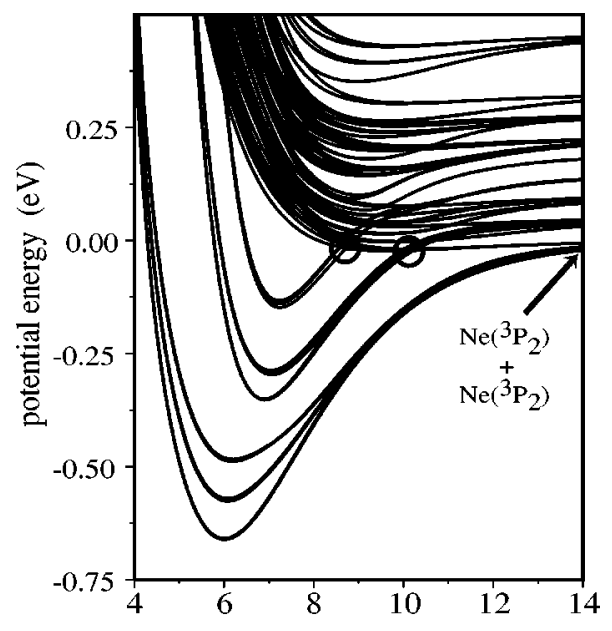

internuclear distance $\mathrm{R}$ (units of $\mathrm{a}_{0}$ )

FIG. 10. Adiabatic potentials for the $\operatorname{Ne}\left\{(2 p)^{5} 3 s\right\}$ $+\operatorname{Ne}\left\{(2 p)^{5} 3 s\right\}$ system including all fine-structure states. The zero point of the energy scale corresponds to the asymptotic energy of the $\mathrm{Ne}\left({ }^{3} P_{2}\right)+\mathrm{Ne}\left({ }^{3} P_{2}\right)$ configuration. Compared to the reduced set of Fig. 1, deeper wells [resembling more closely the $\mathrm{Na}_{2}\left({ }^{1} \Sigma_{g}^{+}\right)$ state] have developed in the states connected asymptotically to $\mathrm{Ne}\left({ }^{3} P_{2}\right)+\mathrm{Ne}\left({ }^{3} P_{2}\right)$, indicating the relaxation of the asymptotic spin-orbit configuration. Crossings of the $\mathrm{Ne}\left({ }^{3} P_{2}\right)+\mathrm{Ne}\left({ }^{3} P_{2}\right)$ system with other asymptotic configurations are indicated as well. Such crossings may lead to reduced ionization suppression by funneling flux onto ionizing potentials.

The ionization rate for polarized atoms was found to increase only slightly with the magnitude of this splitting.

A large magnetic confining field, however, could cause Zeeman splittings greater than the centrifugal barrier present in the $l>0$ quintet states. This would open up new channels for depolarizing collisions that require only a splitting within the quintet states. Given the height of the centrifugal barrier, $5.8 \mathrm{mK}$ for $\mathrm{Ne}$ and $l=2$, such processes should be expected to be fully developed at magnetic fields of approximately 60 G. It may be profitable, therefore, to use the minimum practical trapping field for metastable rare-gas atom traps.

Another factor that we have not taken into account is that curve crossings of the $\mathrm{Ne}\left({ }^{3} P_{2}\right)+\mathrm{Ne}\left({ }^{3} P_{2}\right)$ states can occur with other molecular states from the $\operatorname{Ne}\left\{(n p)^{5}(n+1) s\right\}$ $+\operatorname{Ne}\left\{(n p)^{5}(n+1) s\right\}$ system at some intermediate range. For Ne, it is possible to develop potentials for this full system with the recipe developed in Sec. IV. The result of such a treatment is shown in Fig. 10. In this case, the $\left.\mathrm{Ne}^{3}{ }^{3} P_{2}\right)+$ $\mathrm{Ne}\left({ }^{3} P_{2}\right)$ quintet states indeed cross several states with other asymptotic limits. Closer inspection has taught us that some of these are indeed avoided crossings, with the coupling due to the DD and QQ interactions. At the crossing points, a second-order treatment of the DD interaction in fact no longer suffices. Flux entering on one of the quintet states can now be diverted onto curves that allow ionization to occur at short range. In order for the effect of such crossings to be taken into account, an $R$-dependent treatment of the DD and QQ interactions would be necessary, and in addition a much larger system of coupled equations would have to be solved. We consequently leave such a treatment to a future paper.

\section{ACKNOWLEDGMENTS}

We gratefully acknowledge the support of a Cray Research Grant from Cray Research, Inc., and the Dutch Orga- 
nization for Fundamental Research (NWO)/Dutch National Supercomputing Facilities (NCF). The work of E. J. D. Vredenbregt is made possible by financial support from the Royal Netherlands Academy of Arts and Sciences (KNAW).
Additional support from the Netherlands Foundation for Fundamental Research on Matter (FOM) is also acknowledged. We thank J. G. C. Tempelaars, J. P. J. Driessen, T. H. Bergeman, E. Tiesinga, and C. Orzel for helpful discussions.
[1] K. B. Davis et al., Phys. Rev. Lett. 75, 3969 (1995).

[2] M. H. Anderson et al., Science 269, 198 (1995).

[3] C. C. Bradley, C. A. Sackett, J. J. Tollet, and R. Hulet, Phys. Rev. Lett. 75, 1687 (1995).

[4] K. Huang, Statistical Mechanics, 2nd ed. (Wiley, New York, 1987).

[5] W. Ketterle and N. J. van Druten, Adv. At., Mol., Opt. Phys. 37, 181 (1996).

[6] H. Boesten, A. Moerdijk, and B. Verhaar, Phys. Rev. A 54, R29 (1996).

[7] F. H. Mies, C. J. Williams, P. S. Julienne, and M. Krauss, J. Res. Natl. Inst. Stand. Technol. 101, 521 (1996).

[8] For Cs the situation seems less promising; see, e.g., J. Söding, D. Guéry-Odelin, P. Desbiolles, G. Ferrari, and J. Dalibard, Phys. Rev. Lett. 80, 1869 (1998).

[9] H. Metcalf and P. van der Straten, Phys. Rep. 244, 203 (1994).

[10] Argon, Helium, and the Rare Gases, edited by G. A. Cook (Interscience Publishers, New York, 1961).

[11] M.-O. Mewes et al., Phys. Rev. Lett. 78, 582 (1997).

[12] P. E. Siska, Rev. Mod. Phys. 65, 337 (1993).

[13] H. C. Mastwijk, Ph.D. thesis, Utrecht University, Utrecht, The Netherlands, 1997.

[14] G. V. Shlyapnikov, J. T. M. Walraven, U. M. Rahmanov, and M. W. Reynolds, Phys. Rev. Lett. 73, 3247 (1994).

[15] P. O. Fedichev, M. W. Reynolds, U. M. Rahmanov, and G. V. Shlyapnikov, Phys. Rev. A 53, 1447 (1996).

[16] M. D. Hoogerland et al., Appl. Phys. B: Lasers Opt. 62, 323 (1996); M. R. Doery, E. J. D. Vredenbregt, J. G. C. Tempelaars, H. C. W. Beijerinck, and B. J. Verhaar, Bull. Am. Phys. Soc. 42, 969 (1997).

[17] A large set of references to papers dealing with the properties of neon is given in Ref. [24]. See also Refs. [20,30].

[18] J. Weiner, V. S. Bagnato, S. Zilio, and P. S. Julienne, Rev. Mod. Phys. (to be published).

[19] N. F. Mott and H. S. W. Massey, The Theory of Atomic Collisions, 3rd ed. (Oxford University Press, Oxford, 1971).

[20] T. Y. Chang, Rev. Mod. Phys. 39, 911 (1969).

[21] J. O. Hirschfelder and W. J. Meath, in Nature of Intermolecular Forces, Vol. XII of Advances in Chemical Physics, edited by J. O. Hirschfelder (Interscience Publishers, New York, 1967).

[22] D. Hausamann (private communication).
[23] C. Froese Fischer, The Hartree-Fock Method for Atoms (Wiley, New York, 1977).

[24] M. R. Doery et al., Phys. Rev. A 57, 3603 (1998).

[25] M. Marinescu (private communication).

[26] B. Bussery and M. Aubert-Frécon, J. Chem. Phys. 82, 3224 (1985).

[27] D. D. Konowalow, M. E. Rosencrantz, and M. L. Olson, J. Chem. Phys. 72, 2612 (1980); see also W. T. Zemke and W. C. Stwalley, ibid. 100, 2661 (1994); G. Jeung, J. Phys. B 16, 4289 (1983); A. Henriet and F. Masnou-Seeuws, ibid. 17, 3417 (1984).

[28] D. Hennecart and F. Masnou-Seeuws, J. Phys. B 18, 657 (1985).

[29] D. Hennecart, Ph.D. thesis, Université de Caen, Caen, France, 1982.

[30] S. Fraga and J. Muszyńska, Atoms in External Fields, 2nd. ed. (Elsevier Scientific, New York, 1981).

[31] G. Herzberg, Spectra of Diatomic Molecules, 2nd ed. (Van Nostrand Reinhold, New York, 1950), Vol. I.

[32] D. M. Jones and J. S. Dahler, Phys. Rev. A 37, 2916 (1988).

[33] S. S. Op de Beek, J. P. J. Driessen, H. C. W. Beijerinck, and B. J. Verhaar, J. Chem. Phys. 106, 182 (1997).

[34] S. S. Op de Beek et al., Phys. Rev. A 56, 2792 (1997).

[35] J. Rico, R. López, G. Ramírez, and C. Tablero, J. Chem. Phys. 101, 9807 (1995).

[36] A second important mechanism, the so-called radiative mechanism $[12,33,34]$, does not play a role here since it requires the existence of an electric-dipole-allowed transition to a lower state. By definition, such a transition is absent for metastable atoms.

[37] Semiclassical Methods in Molecular Scattering and Spectroscopy, edited by M. S. Child (Kluwer, Dordrecht, The Netherlands, 1980).

[38] H. Katori, H. Kunugita, and T. Ido, Phys. Rev. A 52, R4324 (1995).

[39] C. Orzel, M. Walhout, U. Storr, P. S. Julienne, and S. L. Rolston (unpublished).

[40] S. J. J. M. F. Kokkelmans and B. J. Verhaar (private communication).

[41] S. Kotochigova, E. Tiesinga, and I. Tupitsyn, Bull. Am. Phys. Soc. 43, 1280 (1998).

[42] R. Gregor and P. E. Siska, J. Chem. Phys. 74, 1078 (1981). 